\title{
On-demand generation of reduced mechanisms based on hierarchically extended intrinsic low-dimensional manifolds in generalized coordinates
}

\author{
Karin König *, Ulrich Maas \\ Institut für Technische Thermodynamik, Universität Karlsruhe (TH), Kaiserstrasse 12, D-76128 Karlsruhe, Germany
}

\begin{abstract}
A new implementation scheme for reduced mechanisms based on hierarchically generated and extended intrinsic low-dimensional manifolds (ILDMs) created "on-demand" is presented. The algorithm includes the use of ILDMs in generalized coordinates and a new hierarchical concept for the extension of the ILDMs into the domain of slow chemistry. Problems of pre-calculated ILDM tables are overcome by generating ILDM cells on-demand during the flame calculation, yielding an increased efficiency of the table generation and implementation. In view of a future generation of ILDMs with adaptive dimension based on a local online error control, the presented algorithm includes the possibility to increase the ILDM dimension hierarchically after the stationary solution (solution after $10^{4} \mathrm{~s}$ ) of the first flame calculation with an $n_{\mathrm{c}}$-dimensional ILDM is reached and to re-calculate the result of this first flame calculation using higher-dimensional manifolds with a subsequent error test. The paper presents the generation of hierarchically extended ILDMs in generalized coordinates as well as the on-demand implementation scheme. A sample free flame calculation for the syngas-air system validates the algorithms.

(C) 2009 The Combustion Institute. Published by Elsevier Inc. All rights reserved.
\end{abstract}

Keywords: Reduced mechanisms; Chemical kinetics; ILDM; Laminar flames

\section{Introduction}

Mainly driven by economical and environmental requirements, the numerical simulation of combustion systems has been subject to increasing interest in the past decades. In these simulations, large numbers of species and reactions have to be considered, with resulting equation systems of high dimension [1]. Furthermore, the calculation of chemical source terms is costly and the chemical kinetics causes a stiffness of

\footnotetext{
Corresponding author. Fax: +49721608 3931.

E-mail address: koenig@itt.mach.uni-karlsruhe.de (K. König).
}

the equation systems [1]. Thus, reduced models to describe the chemical kinetics are an important tool in the context of combustion modeling. Various methods to construct reduced chemical mechanisms (see e.g. [2-4]) have been developed and improved continuosly. Examples are the partial-equilibrium- [5] and steady-state approximations [6], the method of rate-controlled constrained equilibrium (RCCE) [7,8], computational singular perturbation (CSP) [9-16], the method of integral or invariant manifolds (MIM) [17-20], the functional iteration method (FIM) [21-23], flamelet generated manifolds (FGM) [24], trajectory-generated manifolds (TGLDM) [25,26], PIC-/ICE-PIC-Methods [2729] and different concepts for reaction-diffusion

1540-7489/\$ - see front matter (c) 2009 The Combustion Institute. Published by Elsevier Inc. All rights reserved. doi:10.1016/j.proci.2008.05.039 
manifolds (e.g. [30,31]). Another tool for mechanism reduction, which is based on a spectral decomposition of the Jacobian of the chemical source terms is the method of intrinsic lowdimensional manifolds (ILDM) [32,33]. Both theoretical and numerical aspects of the method have been investigated thoroughly (see e.g. [3446,51] and references therein) and the use of ILDMs as pre-calculated lookup-tables in flame calculations has proven to be an efficient tool (e.g. $[47,48])$, though there is a major drawback: typically, no a priori knowledge is used about the part of state space accessed by the flame calculation and the pre-calculated ILDM tables have to cover their whole domain of existence (bounded by the mathematical existence and by additional physical constraints like e.g. nonnegative mass fractions [40]). This is a rather negative aspect in the implementation of pre-calculated tables, because especially laminar flame calculations access only a small part of this tabulated domain. Thus, CPU-time and storage capacity are used unnecessarily to tabulate ILDM points that are not actually needed. An on-demand generation of the tables during the flame calculation can avoid this negative aspect. Therefore, storage and retrieval procedures were suggested like ISAT [49] or PRISM [50], which can in principle be used in combination with different kinds of mechanisms. In [51], an in-situ generation of ILDMs was suggested, showing the potential in saving CPU-time and storage. The algorithm was based on ILDMs of fixed dimension written in terms of species concentrations. The extension of the ILDM to the slow chemistry domain was done by setting the reaction rates of major species to zero. In this paper, we present an algorithm for the ondemand generation of ILDMs in generalized coordinates (generalized coordinates in the ILDM-context are discussed in [44,45]), coupled with a new hierarchical concept for the extension of ILDMs into the slow chemistry domain. Furthermore, the algorithm increases the dimension of the ILDM table automatically after the first flame calculation (note, that "first flame calculation" is here used to refer to the whole calculation with the $n_{\mathrm{c}}$-dimensional ILDM until the stationary solution is obtained, not only to one calculation step), exploiting the hierarchical structure of ILDMs [41]. The higher-dimensional table is then used to re-calculate the result of the first flame calculation with the lower-dimensional table, followed by a subsequent error test. This is a first step towards ILDMs with adaptive dimension controlled by an online error estimation. We present the governing equations (Section 2), the on-demand implementation scheme (Section 3), a sample calculation (Section 4) and give some concluding remarks (Section 5).

\section{Governing equations}

The equations for the calculation of ILDMs have been discussed in detail e.g. in $[40,44,45]$. Let us just recall, that the ILDM of a reaction system governed by

$\frac{\partial \psi}{\partial t}=\boldsymbol{F}(\boldsymbol{\psi})$

can be obtained by solving the $n=\left(n_{\text {spec }}+2\right)$ dimensional ( $n_{\text {spec }}$ : number of species) equation system:

$$
\begin{aligned}
& \boldsymbol{G}(\boldsymbol{\psi})=\widetilde{\boldsymbol{Z}}_{\mathrm{f}}(\boldsymbol{\psi}) \cdot \boldsymbol{F}(\boldsymbol{\psi})=0 \\
& \widetilde{\boldsymbol{P}}(\boldsymbol{\psi})=0 .
\end{aligned}
$$

In Eqs. (1) and (2), $\psi=\left(h, p, w_{1} / M_{1}, \ldots, w_{i} / M_{i}\right)$, $i=1, \ldots, n_{\text {spec }}$, is the $n$-dimensional vector of thermokinetical state variables with $h$ as the enthalpy, $p$ as the pressure; $w_{i} / M_{i}$ are specific mole numbers with $w_{i}$ and $M_{i}$ as mass fraction and molar mass of the species $i$. Symbol $t$ denotes the time and $\boldsymbol{F}(\boldsymbol{\psi})$ the vector-valued function of the chemical rates of change. The $\left(n_{\mathrm{f}} \times n\right)$-dimensional $\left(n_{\mathrm{f}}\right.$ : number of fast processes) left invariant subspace $\widetilde{\boldsymbol{Z}}_{\mathrm{f}}$ is spanning the same space as the "fast" eigenvectors of the Jacobian $\boldsymbol{F}_{\boldsymbol{\psi}}(\boldsymbol{\psi})$. Symbol $\boldsymbol{G}(\boldsymbol{\psi})$ denotes the resulting $n_{\mathrm{f}}$ ILDM-equations and $\boldsymbol{P}(\psi)$ is a vector-valued function of dimension $n_{\mathrm{p}}$ $\left(n_{\mathrm{p}}=n-n_{\mathrm{f}}=n_{\mathrm{z}}+n_{\mathrm{c}}\right.$, where $n_{\mathrm{z}}$ : number of conserved quantities, $n_{\mathrm{c}}$ : number of progress variables) for the parameterization. Details on the parameterization can be found in [40], as well as details on the numerical procedure for the calculation of ILDMs including the multi-dimensional continuation process for the built up of the tabulation mesh.

In the slow chemistry domain (called "SCD" in the following), where the coupling between thermo-chemical and transport processes is strong and the temperature is low, manifolds defined by Eq. (2) require a very high dimension or do not exist. Thus, alternative approaches have to be found for this part of state space. Bykov and Maas [43] suggest a domain-splitting algorithm, in which the extension of the ILDM into the SCD is constructed such that the extended manifold is a locally linear hyperplane, with this hyperplane joining the ILDM boundary with the unburnt point. Tables calculated with this approach yield good results in flame calculations [43] in the SCD, because the processes in this domain are mostly governed by mixing and can therefore be well approximated by a locally linear construction of the extension. Nevertheless, more accurate results would be obtained by an approach that takes into account that there are still some fast chemical processes in the SCD. This can be achieved by a new concept called hierarchically extended ILDMs. In this concept, the extension of an $n_{\mathrm{c}}$-dimensional ILDM is constructed by 
increasing the ILDM dimension stepwise hierarchically by $x=1, \ldots, n_{\mathrm{f}}$ once the boundary of the $n_{\mathrm{c}}$-dimensional ILDM is reached. This dimension increase is done with the additional constraints, that all points of the extension should link the manifold boundary with the unburnt point on the shortest possible path. Concerning the existence of higher-dimensional ILDMs at the boundary of an $n_{\mathrm{c}}$-dimensional ILDM it should be noted, that the existence of an $\left(n_{\mathrm{c}}+1\right)$-dimensional ILDM is guaranteed unless the $\left(n_{\mathrm{c}}+1\right)$ "slowest eigenvalue" degenerates. In this case, an $\left(n_{\mathrm{c}}+2\right)$-dimensional ILDM exists. The movement along the higher-dimensional ILDM causes no problems even in cases of high curvature of the ILDM, because it is calculated using a multi-dimensional continuation procedure [40]. To illustrate the idea of the hierarchical extended ILDMs, Fig. 1 shows a 1d-ILDM (black line) and the point where it ceases to describe the dynamics (black-filled circle) in a schematical 3dprojection of state space. Once the last 1d-ILDM point is reached, the number of ILDM equations used is reduced by one, that means, the dynamics is now moving along a $2 \mathrm{~d}$-manifold (black mesh). The "missing" ILDM equation is replaced by an additional constraint that forces the extension to move towards the unburnt composition (note that the $2 \mathrm{~d}-\mathrm{ILDM}$ is plotted for illustration purpose only, it does not need to be calculated). For a hierarchical extension of a 1d-ILDM, this constraint is simply given by the direction vector defined by the state at the local starting point for the continuation procedure and the unburnt point. In case of an extension of a 2d-ILDM, two different directions would be required to find a surface, that is a subset of the corresponding 3d-ILDM and that links the boundary of the 2d-ILDM with the

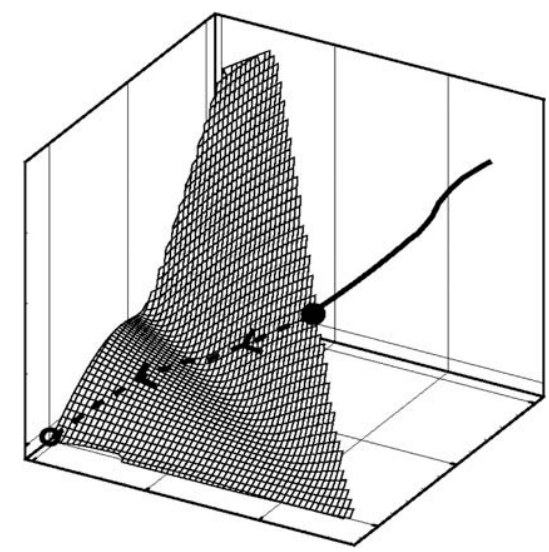

Fig. 1. Illustration of the concept of hierarchically extended ILDMs. 1d-ILDM (black solid line) and point, where the 1d-domain ends (black-filled circle). 2d-ILDM (black mesh) and hierarchical extension of the 1d-ILDM (dashed black line), unburnt point (unfilled circle). unburnt composition. Two directions define this surface, the vector between one node of the last ILDM cell and the unburnt point and one cell vector of this cell. Cell vectors are given as vectors (or linear combinations of those) between the nodes of the ILDM cell; of those vectors we choose the linear combination which has an angle with the vector to the unburnt point closest to $90^{\circ}$. The equation system for the extension, that replaces the ILDM equation system (2) reads:

$$
\begin{aligned}
& \boldsymbol{G}(\boldsymbol{\psi})=\widetilde{\boldsymbol{Z}}_{\mathrm{f}}^{*}(\boldsymbol{\psi}) \cdot \boldsymbol{F}(\boldsymbol{\psi})=0 \\
& \boldsymbol{H}(\boldsymbol{\psi})=\boldsymbol{S}(\boldsymbol{\psi}) \cdot\left(\boldsymbol{\psi}_{\mathrm{ub}}-\boldsymbol{\psi}\right)=0 \\
& \widetilde{\boldsymbol{P}}(\boldsymbol{\psi})=0
\end{aligned}
$$

with $x$ extension equations $\boldsymbol{H}(\boldsymbol{\psi})$ being used instead of $x$ ILDM equations $\boldsymbol{G}(\boldsymbol{\psi})$. Symbol $\widetilde{\boldsymbol{Z}}_{\mathrm{f}}^{*}$ is the fast invariant $\left(\left(n_{\mathrm{f}}-x\right) \times n\right)$-dimensional subspace. The thermokinetical state variables at the unburnt point are included in $\psi_{\mathrm{ub}}$; of course, it can be replaced by any other point in state space to which the extension should be directed, e.g. fuel and oxidizer composition can be specified, if an ILDM should be calculated for the use in a diffusion flame calculation. In this case, the mixture fraction is included in the ILDM as an additional coordinate and for given mixture fractions $\chi$, the corresponding unburnt states are chosen as $\psi_{\mathrm{ub}}(\chi)$. At the last already existing point of the manifold $\psi_{\mathrm{p}}$, the $(x \times n)$-dimensional direction matrix $\boldsymbol{S}(\boldsymbol{\psi})$ is calculated as

$$
\boldsymbol{S}=\left(\begin{array}{c}
\boldsymbol{P} \\
\widetilde{\boldsymbol{Z}}_{\mathbf{f}}^{*} \boldsymbol{F}_{\psi}
\end{array}\right)^{\perp} \cdot\left(\boldsymbol{I}-\boldsymbol{V} \cdot\left(\boldsymbol{V}^{\mathrm{T}} \boldsymbol{V}\right)^{-1} \cdot \boldsymbol{V}^{\mathrm{T}}\right) ;
$$

with $\boldsymbol{S}, \boldsymbol{P}, \widetilde{\boldsymbol{Z}}_{\mathrm{f}}^{*}, \boldsymbol{F}_{\boldsymbol{\psi}}$ and $\boldsymbol{V}$ being functions of $\boldsymbol{\psi}$. Symbol $\boldsymbol{P}$ denotes the $\left(n_{\mathrm{p}} \times n\right)$-dimensional parameterization matrix (details in [40]), $\widetilde{\boldsymbol{Z}}_{\mathrm{f}}^{*} \boldsymbol{F}_{\psi}$ is of dimension $\left(\left(n_{\mathrm{f}}-x\right) \times n\right)$ and $\boldsymbol{V}$ is an $\left(n \times n_{\mathrm{c}}\right)$ dimensional matrix which contains the direction vectors for the extension. It consists of the direction vector linking the last manifold cell and the unburnt composition and of cell direction vectors of the last manifold cell. In this work, we use two steps of the extension, with $x=1$ and $x=n_{\mathrm{f}}$. The boundary, where $x$ has to be increased can be found by a definition following [44,45]: a gap condition defines the boundary, respectively, the existence limit of an $n_{\mathrm{c}}$-dimensional ILDM as $\psi_{\text {boundary }}: \operatorname{Re}\left(\lambda_{n_{\mathrm{c}}} / \lambda_{n_{\mathrm{c}}+1}\right) \geqslant \varepsilon \wedge\left(1 / \tau_{\text {phys }}\right) / \operatorname{Re}\left(\lambda_{n_{\mathrm{c}}+1}\right) \geqslant \varepsilon$ with $\varepsilon$ as a small, user-defined value and $\tau_{\text {phys }}$ as a physical timescale. It should finally be noted, that using $x=n_{\mathrm{f}}$ corresponds to using the approach presented in [43]. Figure 2 illustrates the concept with different ILDMs of the stoichiometric syngas-air system, which have been projected into the $\mathrm{CO}_{2}-\mathrm{H}_{2} \mathrm{O}-\mathrm{H}$-space. Even though the concept of hierarchical extension can be applied to arbitrary ILDM dimensions, we use 1dand 2d-ILDMs here to ensure a clear graphical representation. The 1d-ILDM is shown as black 


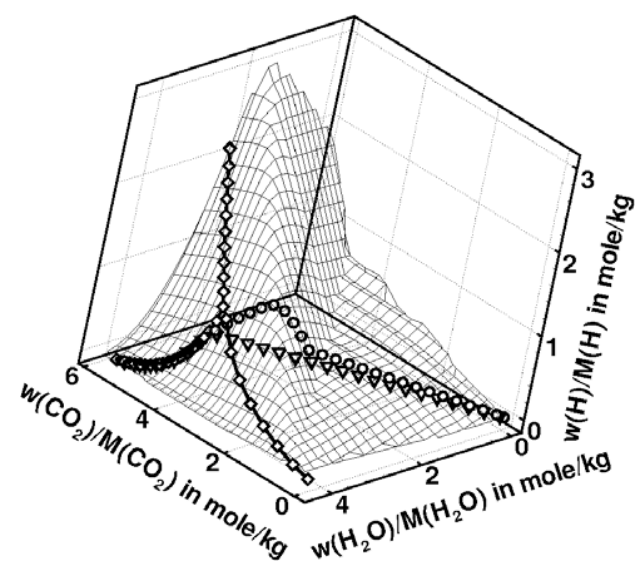

Fig. 2. Projection of different ILDMs into the $\mathrm{CO}_{2-}$ $\mathrm{H}_{2} \mathrm{O}-\mathrm{H}$ space; stoichiometric syngas-air system. 1dILDMs (black without symbols, black with white-filled squares), 1d-ILDM with $\left(x=n_{\mathrm{f}}\right)$-extension (white-filled black triangle symbols), hierarchically extended 1dILDM (white-filled black circles), 2d-ILDM with $\left(x=n_{\mathrm{f}}\right)$-extension (black mesh).

line. Its calculation was stopped at the point, where the solution of the ILDM equation system (2) is not longer unique - from this point on, two different solutions can be found (black lines with white-filled squares). For the extension itself, different approaches have been used: the white-filled black triangles show a 1d-ILDM extended with $x=n_{\mathrm{f}}$, yielding a linear line between the last ILDM and the unburnt point. The open circles represent the same 1d-ILDM which was extended hierarchically. Also shown is the 2d-ILDM of the system (black mesh), which was generated hierarchically and extended with $x=n_{\mathrm{f}}$. It can well be seen, that the $\left(x=n_{\mathrm{f}}\right)$-extension of the 1d-ILDM does not correspond to the 2d-ILDM and is thus only a rough approximation to the dynamics of the system in the part of state space, where a 2d-ILDM is still existing. Opposed to that, the hierarchical extension of the 1d-ILDM lies within the 2dILDM, which makes it a considerably better description of the dynamics of the system. Starting from the boundary of the 2d-ILDM, both the hierarchically extended 1d- and the 2d-ILDM were extended using $x=n_{\mathrm{f}}$, as the locally linear extension showed good results for 2d-ILDMs in [44].

\section{On-demand implementation scheme}

Both the ILDM and its hierarchically generated extension are tabulated in terms of generalized coordinates $[44,45]$, corresponding to mesh coordinates. The mesh, which is build up during the ILDM calculation [40], has an orthogonal structure and is ideally suited to describe the reaction progress. Using the mesh coordinates as tabulation variables is not as intuitive as a tabulation in terms of e.g. certain species or combinations of those and has a permanently changing physical meaning, but it avoids problems in regions, where the tabulation in terms of certain species might be ill-conditioned or might yield a non-unique representation of the ILDM [44]. Also, this kind of tabulation is perfectly suited for an on-demand generation of additional cells. The code used for the flame calculation has to be adapted to the use of ILDMs tabulated in generalized coordinates, which means that the equation system for a reactive flow system [1] has to be re-written in terms of the generalized coordinates $\boldsymbol{\theta}$ (see details in [44]):

$\frac{\partial \boldsymbol{\theta}}{\partial t}=\boldsymbol{\psi}_{\theta}^{+} \boldsymbol{F}(\boldsymbol{\psi})-\boldsymbol{v} \operatorname{grad}(\boldsymbol{\theta})-\frac{1}{\rho} \boldsymbol{\psi}_{\theta}^{+} \boldsymbol{Z}_{s} \widetilde{\boldsymbol{Z}}_{s} \operatorname{div}\left(\boldsymbol{D} \boldsymbol{\psi}_{\theta} \operatorname{grad}(\boldsymbol{\theta})\right)$.

Symbol $\psi_{\theta}^{+}$is the Moore-Penrose pseudo-inverse [52] of $\psi_{\theta} ; \boldsymbol{Z}_{\mathrm{s}}$ is the slow invariant subspace of $\boldsymbol{F}_{\boldsymbol{\psi}}(\boldsymbol{\psi})$ and $\boldsymbol{Z}_{\mathrm{s}}$ is the inverse of $\boldsymbol{Z}_{\mathbf{s}}$. The variables $\psi, \psi_{\theta}, D, Z_{\mathrm{s}}$ and $\widetilde{\boldsymbol{Z}}_{\mathrm{s}}$ are functions of the generalized coordinate $\boldsymbol{\theta}$ only and can therefore be calculated on each mesh point of the ILDM and its extension independently from the flame calculation.

In this work, we use a new on-demand implementation scheme for the hierarchically extended ILDMs in generalized coordinates. Only a small starting ILDM table is calculated beforehand, allowing the flame calculation to generate a starting profile. Once the flame calculation has been started, needed values are interpolated from the ILDM table and if they cannot be found, the following algorithm is used to add the needed cells:

(1) Transform generalized coordinates requested by the flame calculation into vertex coordinates for the cell to be generated.

(2) Search the table for already existing neighboring cells.

(3) Check, whether new cell is in the ILDM- or extension-domain.

(4) Generate the new cell using either ILDMor extension-equations.

(5) Check, whether there are unclosed cells and if yes, close them.

(6) Check the manifold for convexity and add cells, if necessary to reach convexity. For a discussion of manifold convexity see [53].

(7) Calculate the transport data for Eq. (5).

(8) Return extended table and proceed with flame calculation.

After the flame calculation is finished, the ondemand calculated ILDM is adapted to the calculation and includes only cells - except maybe some 
of the starting cells - that are really needed during the calculation. Therefore, the on-demand ILDM generation according to the steps 1-8 overcomes the drawback of too large tabulated domains in pre-calculated ILDM tables. Nevertheless, there is another problem appearing when pre-calculated tables are used, namely the fixed table dimension, that has to be defined for the table setup before the flame calculation - mostly with no sufficient a priori knowledge, which dimension suits best. Previous implementations did neither offer the possibility of a dimension change for the whole table, nor of a local change of the dimension in a single computational cell in the flame calculation. Of course, the latter would be the best solution: a full adaptivity of the dimension of the table, corresponding to the local situation at the momentarily calculated grid point and time step. Implementing such fully adaptive tables is possible, but yields complex procedures e.g. for storage organization. A first step towards such an adaptive dimension is to change the dimension of the whole table and to calculate the flame based on two different table dimensions. Our algorithm includes this possibility, with the following steps being performed after the first flame calculation based on an $n_{\mathrm{c}}$-dimensional ILDM is finished:

(A) Delete the $\left(x=n_{\mathrm{f}}\right)$-part of the hierarchical extension from the $n_{\mathrm{c}}$-dimensional table. This is necessary, as it has not been proven yet, whether a hierarchical dimension increase can be applied for this part of the extension. The points with $x=1$ and the points of the $n_{\mathrm{c}}$-dimensional ILDM remain in the table, as they are already points of the $\left(n_{\mathrm{c}}+1\right)$-dimensional ILDM.

(B) Generate the $\left(n_{\mathrm{c}}+1\right)$-dimensional ILDM hierarchically [41] with the table being restricted to a small domain only.

(C) Add the $\left(x=n_{\mathrm{f}}\right)$-extension to the new ILDM.

(D) Calculate the necessary data for Eq. (5) (transport matrix, projection matrix, reduced source terms) and pass the new $\left(n_{\mathrm{c}}+1\right)$-dimensional table to the flame calculation.

(E) Re-start the flame calculation using the $\left(n_{\mathrm{c}}+1\right)$-dimensional ILDM-table; the solution of the previous calculation with the $n_{\mathrm{c}}$ dimensional table is used as starting profile for faster convergence.

(F) Generate further cells on-demand following steps $1-8$, if necessary.

(G) Calculate the error to show the effect of increasing the dimension of the table.

The error test in step $\mathrm{G}$ is performed by calculating the relative error between the solutions obtained for the $n_{\mathrm{c}^{-}}$and the $\left(n_{\mathrm{c}}+1\right)$-dimensional
ILDM (or the solutions for an $n_{\mathrm{c}}$-dimensional ILDM and the detailed solution) with the relative error for a species $i$ being defined by

$$
\epsilon_{\mathrm{rel}, i}=\frac{\left|\boldsymbol{\psi}_{i}^{n_{\mathrm{c}}+1}-\boldsymbol{\psi}_{i}^{n_{\mathrm{c}}}\right|}{\max \left(\alpha,\left|\boldsymbol{\psi}_{i}^{n_{\mathrm{c}}+1}\right|\right)}
$$

with $\alpha$ as a lower error threshold.

With the presented algorithm, it is possible to calculate the flame based on different table dimensions and to compare the results, what can be considered a first step towards an optimization of the used table dimension without the need for an a priori knowledge about the ideal dimension.

\section{Sample calculations}

A sample 1d-calculation for a free, adiabatic, premixed syngas-air flame in a laminar flow field is presented to validate the algorithms. For such validation purposes, the syngas-air system is a good reference system and it has been discussed in previous publications on ILDM (e.g. [44] and references therein). Also, the system is simple (13 species, 67 elementary reactions), but nevertheless a representative test case, showing all characteristics of a typical combustion system. We consider a stoichiometric mixture with an unburnt temperature of $298 \mathrm{~K}$ at a constant pressure of $1 \mathrm{bar}$. A simplified transport model (equal diffusivities, Lewis number equal to unity) is used (for complex transport models in the ILDM-context see [35]; for the case of non-equal diffusivities see $[44,45]$ ). It should be noted, that the described on-demand algorithm does not depend on neither the system simplicity nor on the use of simplified transport models. First, the different steps of the algorithms shall be discussed. Figure 3 illustrates the flame calculation with a 1d-ILDM and the subsequent automatical hierarchical generation of a 2d-table. Part (a) of the figure shows the starting ILDM for the first flame calculation, a hierarchically extended 1d-ILDM (black line). Also shown is the starting profile of the flame calculation (dashed black line); it is generated by linking burnt and unburnt composition by a straight line in detailed variables and projecting this line onto the reduced variables. The stationary solution (solution after $10^{4} \mathrm{~s}$ ) of the flame calculation (square symbols) lies on the 1d-ILDM. Of course, the solution for a 1d-ILDM is a good approximation only in a very small part of state space, but it should be noted, that the computational results provided by the hierarchically extended 1d-ILDM are in better agreement with the detailed solution (triangle symbols) than those obtained for an ILDM extended with $x=n_{\mathrm{f}}$ (dash-dotted black line). Then, the 1d-ILDM is used to automatically generate a 2d-ILDM according to steps A-G. Part (b) of the figure shows the remaining ILDM after 

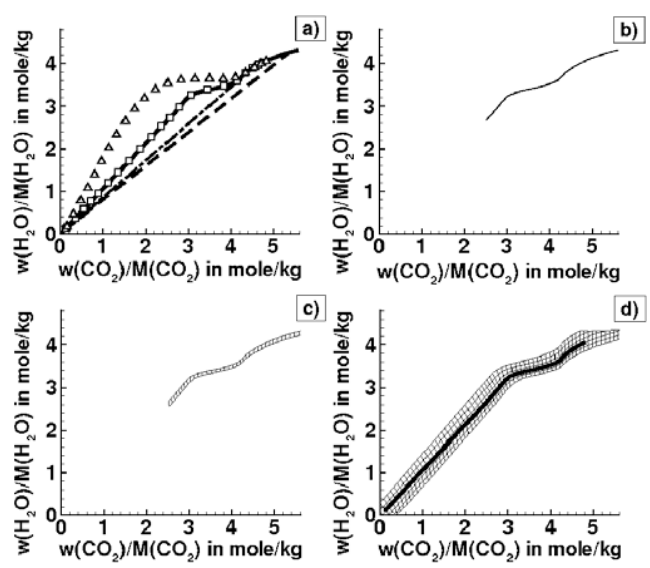

Fig. 3. Projections of state space into the $\mathrm{CO}_{2}-\mathrm{H}_{2} \mathrm{O}-$ space; stoichiometric syngas-air system. (a) Hierarchically extended 1d-ILDM (solid black line), starting profile (dashed black line) and stationary solution (square symbols) of the free flame calculation. Stationary solution of the free flame calculation for a $\left(x=n_{\mathrm{f}}\right)$ extended ILDM (dash-dotted black line). Solution of the detailed system (triangle symbols). (b) 1d-ILDM after removal of the $\left(x=n_{\mathrm{f}}\right)$-extension. (c) Hierarchically generated 2d-ILDM. (d) 2d-ILDM with $\left(x=n_{\mathrm{f}}\right)$-extension (black mesh) and starting solution for the second flame calculation (black line, corresponds to stationary solution of the first calculation).

step A, corresponding to the 1d-ILDM and its hierarchical extension with $x=1$ and Part (c) shows the hierarchically generated 2d-ILDM after step B. Note, that the hierarchically extended 1dILDM is better suited for the hierarchical generation than a non-extended 1d-ILDM: the points of the $(x=1)$-extension are elements of the $2 \mathrm{~d}$ ILDM and therefore, the hierarchically extended ILDM increases the number of already known points, yielding increased efficiency and stability of the hierarchical generation. Following the hierarchical generation, some more cell rows are added to the ILDM to provide enough cells for the first integrations during the second flame calculation (this means the whole calculation done with the $\left(n_{\mathrm{c}}+1\right)$-dimensional ILDM) and to increase the stability of the extension algorithm. Afterwards, the $\left(x=n_{\mathrm{f}}\right)$-extension is added to the ILDM, with the result being shown in Part (d) of the figure (black mesh). The black line is the starting profile for the second flame calculation based on the 2d-ILDM; it corresponds to the stationary solution obtained from the first flame calculation with the 1d-ILDM (used for faster convergence). Figure 4 illustrates the steps of the second flame calculation with the 2d-ILDM, including the on-demand addition of cells according to the steps 1-8. Part (a) shows the 2d-ILDM (black mesh) after the first cell has been added ondemand at around $8 \cdot 10^{-6} \mathrm{~s}$ after the flame calculation has been started; the black lines show the
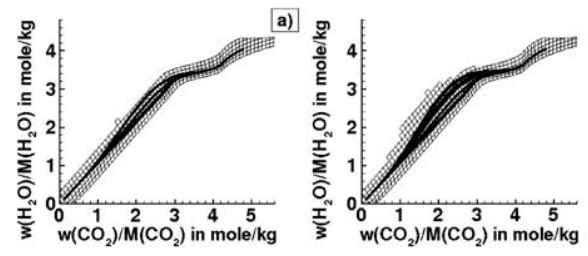

b)
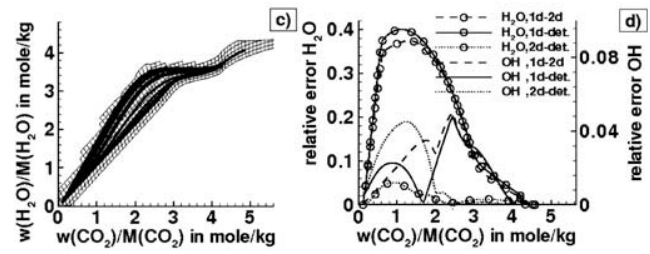

Fig. 4. Projections of state space into the $\mathrm{CO}_{2}-\mathrm{H}_{2} \mathrm{O}$ space in (a)-(c); stoichiometric syngas-air system. (a) $2 \mathrm{~d}$ ILDM after first cell was added on-demand (black mesh) and flame trajectories (black lines), $t \leqslant 8 \cdot 10^{-6} \mathrm{~s}$. (b) On-demand extended 2d-ILDM (black mesh) and flame trajectories (black lines), $t \leqslant 2 \cdot 10^{-5} \mathrm{~s}$. (c) On-demand extended 2d-ILDM (black mesh) and flame trajectories (black lines) after stationary solution has been reached. (d) Relative errors between 1d- and 2d-ILDM based (dashed lines), 1d-ILDM based and detailed (solid lines) and 2d-ILDM based and detailed (dash-dotted line) calculation for species $\mathrm{H}_{2} \mathrm{O}$ (lines with circle symbols) and $\mathrm{OH}$ (lines without symbols), plotted over $\mathrm{CO}_{2}$.

temporal evolution of the flame solution. It can be seen, that the hierarchical generated 2d-ILDM is well suited to the reaction progress which makes it an optimal starting manifold for the on-demand addition. All added cells are calculated automatically in a direction, that suits the reaction progress and in most cases, the cell addition has to be performed in only one direction. To generate the cells, the parameterization of the already existing neighbouring cells can be used (with an update, if enough information is given [40]), which makes the cell calculation efficient and yields a mesh, that is optimally adjusted to the dynamics of the system and that contains cells of uniform structure. Such an optimal structure of the mesh can only be guaranteed by an on-demand generation based on a hierarchical concept. Part (b) of the figure shows the further on-demand cell generation and evolution of the flame trajectories after $2 \cdot 10^{-5} \mathrm{~s}$. "Trajectory" is here used for the solution of a flame calculation in state space at a certain time. The figure again shows the optimal direction of the on-demand generated cells with the mesh following the reaction progress. The final ILDM (after a stationary solution of the flame calculation is obtained) can be seen in Part (c) of the figure together with the flame trajectories; the ILDM does only contain the needed cells and is perfectly adapted to the part of state space accessed by the flame trajectories. In Part (d), the relative errors calculated in step $G$ are shown for the species $\mathrm{H}_{2} \mathrm{O}$ (lines with circle symbols) and $\mathrm{OH}$ (lines without symbols), with the errors comparing the 
1d- and 2d-ILDM based results (dashed) and the 1d-ILDM, respectively, 2d-ILDM based results with the detailed solution (solid and dash-dotted). It can well be seen, that the dimension increase yields a considerable improvement of the obtained stationary solutions. Compared to the solution of the detailed system, the 2d-ILDM based calculation provides good results with small relative errors. Such comparisons between detailed and reduced results are well known from previous publications (see e.g. the ILDM literature cited in Section 1 and references therein), they will therefore be not discussed in detail here (see [45] for the effect of a further dimension increase from 2 to 3). Finally, to illustrate the efficiency of the algorithm, Fig. 5 shows a comparison between a full syngas-air ILDM-table (thin black mesh) and the on-demand extended table (white-filled black mesh). Additionally, the hierarchically extended 1d-ILDM is plotted as black line; all ILDMs are being shown as projections into the $\mathrm{CO}_{2}-\mathrm{H}_{2} \mathrm{O}-\mathrm{OH}$-space. It can clearly be seen, that the on-demand generated table covers only a small part of the full table; with 427 cells being contained in the on-demand, 2691 cells in the full table. Thus, the saving in storage capacity is accordingly high, as it is reduced by $82 \%$ in this example. Further reduction of the storage capacity can be achieved by deleting already used cells. The algorithm results in a saving of $\approx 22 \% \mathrm{CPU}$ time in the discussed example (compared to the CPU-time needed for the generation of a full 1dand a full 2d-ILDM-table and flame calculations with these tables). Note, however, that in the

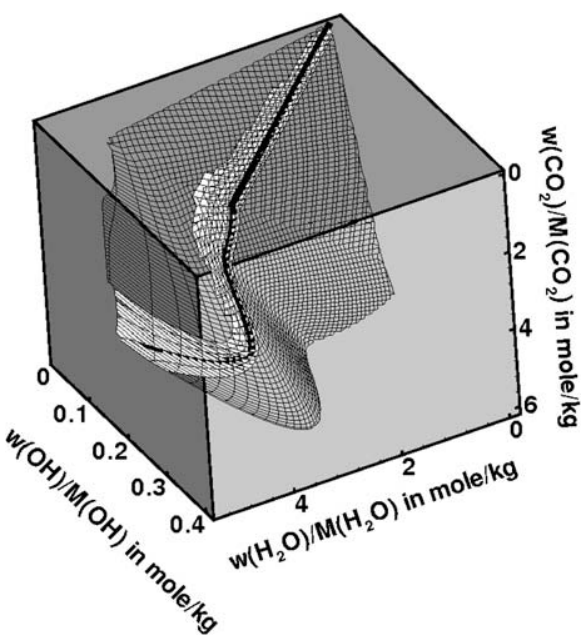

Fig. 5. Projection of state space into the $\mathrm{CO}_{2}-\mathrm{H}_{2} \mathrm{O}-\mathrm{OH}-$ space, stoichiometric syngas-air system. 2d-ILDM with $\left(x=n_{\mathrm{f}}\right)$-extension covering the whole domain (thin black mesh), 2d-ILDM after on-demand generation (white-filled black mesh) during the free flame calculation. Hierarchically extended 1d-ILDM (black line). one-dimensional case a model reduction is not needed, because the flame calculation can be done with detailed mechanisms at low computational cost. The field of the proposed algorithm is in the simulation of multi-dimensional flames, where the computational effort will be reduced considerably.

\section{Conclusions}

An algorithm for the on-demand generation of hierarchically extended and hierarchically generated ILDMs in generalized coordinates has been presented. An approach for the hierarchical extension of ILDMs into the domain of slow chemistry is discussed which does not only increase the accuracy of calculation results, but also provides a good basis for a subsequent hierarchical generation of a higher-dimensional ILDM. Both the ILDM and its extension are being tabulated in terms of generalized coordinates and are therefore optimally suited for the on-demand addition of cells during the flame calculation. The on-demand generation itself is an efficient way of implementing ILDM tables in flame calculations, as only the actually accessed part of state space is tabulated and therefore, CPU-time and storage capacity can be saved. By exploiting the hierarchical structure of ILDMs to increase the dimension of the ILDM table to $\left(n_{\mathrm{c}}+1\right)$ after the stationary solution of a first flame calculation based on an $n_{\mathrm{c}}$-dimensional ILDM has been reached, the algorithm provides the possibility to re-calculate the result of the first calculation using a higher-dimensional table. A subsequent error test allows to estimate the benefit of the dimension increase. The use of a hierarchically generated ILDM for the second calculation ensures, that the table is ideally adjusted to the reaction progress and increases the efficiency of the on-demand cell generation. Sample calculations for a free syngas-air flame validate the algorithm and show the potential of hierarchically extended ILDMs as well as of the on-demand algorithm combined with hierarchically generated ILDMs. The algorithms can be used for systems and ILDMs of arbitrary dimension and it should be noted, that the efficiency of the algorithms increases with increasing table dimension and system complexity. The algorithm can e.g. be applied for partially premixed turbulent flames. Future work focuses on a fully adaptive implementation of ILDM tables based on an online error control, which would allow to adjust the ILDM dimension locally during the calculation. In the region of slow chemistry, the new REDIM-method [31] could be implemented instead of the hierarchical extension in order to further increase the accuracy of the results. 


\section{Acknowledgement}

The authors express their thanks to the Deutsche Forschungsgemeinschaft for their financial support.

\section{References}

[1] J. Warnatz, U. Maas, R.W. Dibble, Combustion. Physical and Chemical Fundamentals, Modeling and Simulation, Experiments, Pollutant Formation, Springer-Verlag, Berlin, Heidelberg, 2001.

[2] A.S. Tomlin, T. Turanyi, M.J. Pilling, in: M.J. Pilling (Ed.), Mathematical Tools for the Construction, Investigation and Reduction of Combustion Mechanisms, Elsevier, Amsterdam, 1997, pp. 293437.

[3] M.D. Smooke, Lecture Notes in Physics, vol. 384. Springer, New York, 1991.

[4] Z. Ren, S.B. Pope, Combust. Flame 147 (4) (2006) 243-261.

[5] F. Williams, Combustion Theory, Addison-Wesley, New York, 1985.

[6] E. Hesstvedt, O. Hov, I. Isaksen, Int. J. Chem. Kinet. 10 (1978) 971-994.

[7] J.C. Keck, Prog. Eng. Combust. Sci. 16 (2) (1990) $125-154$.

[8] Q. Tang, S.B. Pope, Combust. Theory Model. 8 (2) (2004) 255-279.

[9] S.H. Lam, D.A. Goussis, Proc. Combust. Inst. 22 (1988) 931-941.

[10] D.A. Goussis, S.H. Lam, Proc. Combust. Inst. 24 (1992) 113-120.

[11] S.H. Lam, Combust. Sci. Technol. 89 (5) (1993) 375404.

[12] A. Massias, D. Diamantis, E. Mastorakos, D.A. Goussis, Combust. Flame 117 (4) (1999) 685-708.

[13] T. Lu, Y. Ju, C.K. Law, Combust. Flame 126 (1-2) (2001) 1445-1455.

[14] M. Valorani, F. Creta, D.A. Goussis, J.C. Lee, H.N. Najm, Combust. Flame 146 (1-2) (2006) 2951.

[15] S.H. Lam, Combust. Sci. Technol. 179 (4) (2007) 767-786.

[16] J.C. Lee, H.N. Najm, S. Lefantzi, et al., Combust. Theory Model. 11 (1) (2007) 73-102.

[17] A.N. Gorban, I.V. Karlin, Chem. Eng. Sci. 58 (21) (2003) 4751-4768.

[18] N.N. Bogolyubov, Y.A. Mitropolsky, Asymptotic Methods in the Theory of Nonlinear Oscillations, N.Y. Gordon and Breach, 1961.

[19] N. Fenichel, J. Differ. Equations 31 (1) (1979) 53-98.

[20] V. Goldshtein, V. Sobolev, AMS Transl. Ser. 2153 (1992) 73-92.

[21] S.J. Fraser, J. Chem. Phys. 88 (8) (1988) 4732-4738.

[22] M.R. Roussel, S.J. Fraser, J. Chem. Phys. 93 (2) (1990) 1072-1081.

[23] M.R. Roussel, S.J. Fraser, J. Phys. Chem. 97 (31) (1993) 8316-8327.

[24] J.A. van Oijen, L.P.H. de Goey, Combust. Sci. Technol. 161 (1) (2000) 113-137.
[25] U. Maas, S.B. Pope, FDA 93-11, Cornell University, 1993.

[26] M.J. Davis, R.T. Skodje, J. Chem. Phys. 111 (3) (1999) 859-874.

[27] Z. Ren, S.B. Pope, Proc. Combust. Inst. 30 (2005) $1293-1300$.

[28] Z. Ren, S.B. Pope, A. Vladimirsky, J.M. Guckenheimer, J. Chem. Phys. 124 (11) (2006) 114111.

[29] Z. Ren, S.B. Pope, A. Vladimirsky, J.M. Guckenheimer, Proc. Combust. Inst. 30 (2007) 473-481.

[30] M.J. Davis, J. Phys. Chem. A 110 (16) (2006) 5235 5256, 5257-5272.

[31] V. Bykov, U. Maas, Combust. Theory Model. 11 (6) (2007) 839-862.

[32] U. Maas, S.B. Pope, Combust. Flame 88 (3-4) (1992) 239-264.

[33] U. Maas, S.B. Pope, Proc. Combust. Inst. 24 (1992) $103-112$.

[34] R.T. Skodje, M.J. Davis, J. Phys. Chem. A 105 (45) (2001) 10356-10365.

[35] U. Maas, S.B. Pope, Proc. Combust. Inst. 25 (1994) $1349-1356$

[36] T. Blasenbrey, U. Maas, Proc. Combust. Inst. 28 (2000) 1623-1630.

[37] R.L.G.M. Eggels, L.P.H. de Goey, Combust. Flame 100 (4) (1995) 559-570.

[38] O. Gicquel, D. Thevenin, M. Hilka, N. Darabiha, Combust. Theory Model. 3 (3) (1999) 479-502.

[39] J. Nafe, U. Maas, Proc. Combust. Inst. 29 (2002) $1379-1385$.

[40] U. Maas, Comput. Vis. Sci. 1 (1998) 69-82.

[41] J. Nafe, U. Maas, Combust. Flame 135 (1-2) (2003) $17-26$.

[42] K. König, U. Maas, Proc. Combust. Inst. 30 (2005) $1317-1323$

[43] V. Bykov, U. Maas, Proc. Combust. Inst. 31 (2007) 465-472.

[44] V. Bykov, U. Maas, Proc. 20th ICDERS, P244, Montreal, Canada, 2006.

[45] J. Bauer, V. Bykov, U. Maas, in: P. Wesseling, E. Oñate, J. Périaux (Eds.), Proc. ECCOMAS CFD, No. 475, Egmond aan Zee, The Netherlands, 2006, available at $<$ http://proceedings.fyper.com/ecco mascfd2006/>, ISBN 90-9020970-0.

[46] Z. Ren, S.B. Pope, Combust. Theory Model. 11 (5) (2007) 715-739.

[47] P.A. Nooren, H.A. Wouters, T.W.J. Peeters, D. Roekaerts, U. Maas, D. Schmidt, Combust. Theory Model. 1 (1) (1997) 79-96.

[48] B. Naud, B. Merci, D. Roekaerts, D. Schmidt, U. Maas, in: K. Hanjalic, Y. Nagano, S. Jakirlic (Eds.), Proc. 5th Symp. Turbulence, Mass and Heat Transfer, Croatia, Begell House, 2006.

[49] S.B. Pope, Combust. Theory Model. 1 (1) (1997)41-63.

[50] S.R. Tonse, N.W. Moriarty, N.J. Brown, M. Frenklach, Isr. J. Chem. 39 (1999) 97-106.

[51] C. Correa, H. Niemann, B. Schramm, J. Warnatz, Proc. Combust. Inst. 28 (2000) 1607-1614.

[52] G.H. Golub, C.F. van Loan, Matrix Computations, The John Hopkins University Press, Baltimore and London, 1996.

[53] U. Maas, Proc. 1st. MIT Conference, Elsevier, Amsterdam, 2001, pp. 1304-1308. 